\title{
Percolation of aligned rigid rods on two-dimensional triangular lattices
}

\author{
P. Longone (1), P. M. Centres, and A. J. Ramirez-Pastor* \\ Departamento de Física, Instituto de Física Aplicada, Universidad Nacional de San Luis-CONICET, \\ Chacabuco 917, D5700BWS San Luis, Argentina
}

(Received 15 May 2019; revised manuscript received 6 September 2019; published 4 November 2019)

\begin{abstract}
The percolation behavior of aligned rigid rods of length $k$ ( $k$-mers) on two-dimensional triangular lattices has been studied by numerical simulations and finite-size scaling analysis. The $k$-mers, containing $k$ identical units (each one occupying a lattice site), were irreversibly deposited along one of the directions of the lattice. The connectivity analysis was carried out by following the probability $R_{L, k}(p)$ that a lattice composed of $L \times L$ sites percolates at a concentration $p$ of sites occupied by particles of size $k$. The results, obtained for $k$ ranging from 2 to 80, showed that the percolation threshold $p_{c}(k)$ exhibits a increasing function when it is plotted as a function of the $k$-mer size. The dependence of $p_{c}(k)$ was determined, being $p_{c}(k)=A+B /(C+\sqrt{k})$, where $A=p_{c}(k \rightarrow \infty)=0.582(9)$ is the value of the percolation threshold by infinitely long $k$-mers, $B=$ $-0.47(0.21)$, and $C=5.79(2.18)$. This behavior is completely different from that observed for square lattices, where the percolation threshold decreases with $k$. In addition, the effect of the anisotropy on the properties of the percolating phase was investigated. The results revealed that, while for finite systems the anisotropy of the deposited layer favors the percolation along the parallel direction to the alignment axis, in the thermodynamic limit, the value of the percolation threshold is the same in both parallel and transversal directions. Finally, an exhaustive study of critical exponents and universality was carried out, showing that the phase transition occurring in the system belongs to the standard random percolation universality class regardless of the value of $k$ considered.
\end{abstract}

DOI: 10.1103/PhysRevE.100.052104

\section{INTRODUCTION}

Percolation is a very active field of research and applied to a wide range of fields, such as biology, nanotechnology, device physics, physical chemistry, and materials science [1-4]. The problem of percolation is not a new one but still attracts considerable interest [5-7], and some unsolved questions remain.

Percolation theory was derived for periodic lattices of sites (bonds) which are occupied with probability $p$ or empty (nonoccupied) with probability $(1-p)[1]$. In the case of deposition processes, $p$ coincides (in the thermodynamic limit) with the coverage of the lattice or fraction of sites occupied by the deposited objects. If the concentration of these objects is sufficiently large, a cluster (a group of occupied sites in such a way that each site has at least one occupied nearest-neighbor site) extends from one side to the opposite one of the system. The central idea of the percolation theory is based on finding the minimum concentration for which a complete path of adjacent sites crossing the entire system becomes possible. This value of the concentration rate is named the critical concentration or percolation threshold $p_{c}$ and determines the phase transition in the system [1].

One of the most popular methods of studying percolation of deposited objects is the random sequential adsorption (RSA) technique [8-11]. In this process, objects of a specified shape are randomly and sequentially adsorbed onto a substrate

*Corresponding author: antorami@unsl.edu.ar and then immobilized. Excluded volume, or particle-particle interaction, is incorporated by rejection of deposition overlap, while particle-substrate interaction is modeled by the irreversibility of deposition. The final state generated by RSA is a disordered state (known as jamming state), in which no more objects can be deposited due to the absence of free space of appropriate size and shape (the jamming state has infinite memory of the process and the orientational order is purely local). Thus, a competition between percolation and jamming is established $[9,10]$. In some applications one may want that percolation dominates (e.g., communications), and in others one may prefer that jamming dominates and percolation is suppressed at an early stage (e.g., forest fires).

From an experimental point of view, RSA model and its variants have been widely studied to explain the observations of various natural and experimental scenarios [12-19]. For example, the model has been used to describe adsorption phenomena of proteins at solid-liquid interfaces [12-17]. In the absence of significant protein-protein interactions, except short-range repulsions, proteins populate the surface in a purely randomized arrangement, which is described by the RSA theory. According to this framework proteins only adsorb to the surface if they approach an area which does not overlap with any other pre-adsorbed protein. Otherwise, if a protein were to hit an adsorbed protein on its way toward the surface, it is rejected back into the bulk volume. This ends up in a very inefficiently packed protein layer leaving undefined gaps between adsorbed proteins which are not large enough to accommodate another protein. RSA process has been also 
applied to model the microstructure of composites containing randomly oriented fibers $[18,19]$.

For randomly distributed and isotropically oriented linear $k$-mers [20] (linear rigid particles occupying $k$ consecutive sites) on square lattices, it was shown that the percolation threshold does not change monotonically with the length of needles [21-23]. For short objects the percolation threshold decreases rapidly, goes through a minimum around $k=$ $13, \ldots, 15$, and then starts to increase moderately. Later Kondrat et al. [24] presented a strict proof that in any jammed configuration of nonoverlapping, fixed-length, horizontal or vertical needles on a square lattice, all clusters are percolating clusters. The theorem refutes the conjecture [21-23] that in the RSA of such needles on a square lattice, percolation does not occur if the needles are longer than some threshold value $k^{*}$, estimated to be of the order of several thousand.

In a very recent paper, Slutskii et al. [25], using simulation techniques, corroborated the result reported by Kondrat et al. [24]. Based in a very efficient parallel algorithm, the authors studied the problem of large linear $k$-mers (up to $k=2^{17}$ ) on a square lattice with periodic boundary conditions. The obtained results indicate that the percolation threshold tends to a constant value as $k \rightarrow \infty$, being $p_{c}(k \rightarrow \infty)=0.615(1)$. The limit value of $p_{c}$ is lower than the asymptotic value of the jamming coverage: $p^{j}(k \rightarrow \infty)=0.655(9)$ [26]. This finding reinforces the theoretical analysis in Ref. [24], namely, in the case of linear $k$-mers on square lattices, percolation always occurs before jamming.

An interesting problem arises when the probability of taking horizontal and vertical orientation is not the same. In this context, the advent of modern techniques for building highly conductive rodlike particles (such as carbon nanotubes [27], metal nanowires [28], etc.) has considerably encouraged the investigation of anisotropic composites made of these elongated particles on an insulating matrix. The study of the conductive properties of these composite materials is an area of increasing interest for the production of flexible transparent conductors [29-31], with diverse applications in solar cells, touch screens, and transparent heaters [32-36]. These promising applications are inspiring both theoretical and experimental studies in this field [37].

In order to design a composite with the desired properties, it is crucial to understand and control the formation of a system-spanning network of nanofillers inside the host matrix, which happens above a critical concentration of filler material. This critical concentration coincides with the percolation threshold of the system [38,39], demonstrating the importance of percolation theory and its applicability to studying the electrical conductivity of materials composed of rodlike highly conducting fillers. Along this line, numerous works have been conducted on percolation of rodlike particles and its connection with the electrical conductivity [40-47]. The studies in Refs. [40-47] represent an important step in the understanding of the percolating properties of anisotropic conductors.

For the continuum problem, several theoretical and simulation studies have been performed on the impact of particle alignment on percolation in three-dimensional space. These studies showed (1) that percolation happens along the alignment direction and perpendicular to that at the same concentration and (2) that the probability of percolation crosses at a value different from 0.5 for different system sizes, depending also on the probing direction. Relevant literature about this subject can be found in Refs. [48-54].

In the case of lattice models, which is the topic of this paper, the effect of anisotropy (or $k$-mer alignment) on percolation was recently investigated for the case of aligned rigid $k$-mers on square lattices [55]. The results, obtained for $k$ ranging from 1 to 14 , showed that (1) the percolation threshold exhibits a decreasing function when it is plotted as a function of the $k$-mer size and (2) for any value of $k(k>1)$, the percolation threshold is higher for aligned rods than for rods isotropically deposited. Later Tarasevich et al. [21] extend the analysis in Ref. [55] to larger lattices $(100 \leqslant L \leqslant 19200)$ and longer objects $(2 \leqslant k \leqslant 512)$. The authors corroborate the results obtained by Longone et al. [55] for the case of perfectly aligned rods and complete the study by including the percolation behavior of partially ordered phases (states whose degree of anisotropy varies between the two limit cases, i.e., isotropic and perfectly aligned $k$-mers).

Interesting results have also been reported on triangular lattices: RSA of objects of various shape [56], reversible RSA [57], reversible RSA of mixtures [58], anisotropic RSA of extended objects [59], percolation of extended objects [60], and jamming and percolation in RSA of extended objects on lattice with quenched impurities [61]. However, the effect of $k$-mer alignment on percolation has been poorly studied. In this context, the main objective of the present paper is to study the percolation behavior of aligned rigid rods on 2D triangular lattices. For this purpose, extensive numerical simulations (with $2 \leqslant k \leqslant 80$ and $75 \leqslant L / k \leqslant 640$ ) supplemented by analysis using finite-size scaling theory have been carried out. The obtained results revealed that the percolation threshold $p_{c}(k)$ is an increasing function with $k$. This finding contrasts with the decreasing tendency observed for $p_{c}(k)$ in square lattices, showing that (1) it is of interest and of value to inquire how a specific lattice structure influences the main percolation properties of particles occupying more than one site and (2) the structure of the lattice plays a fundamental role in determining the statistics of extended objects. In addition, the anisotropy effect on the percolation probabilities characterizing the different lattice directions was investigated. The study also includes a complete analysis of critical exponents and universality.

The present work is a natural extension of our previous research in the area of percolation of polyatomic species, and the results obtained here could have potential application in the field of conductivity in composite materials. The paper is organized as it follows: the model and basic definitions are given in Sec. II. Percolation properties are studied in Sec. III. Finally, the conclusions are drawn in Sec. IV.

\section{MODEL AND BASIC DEFINITIONS}

Straight rigid rods are deposited randomly, sequentially, and irreversibly on a $2 \mathrm{D}$ triangular lattice. In the computer simulations, a rhombus-shaped system of $M=L \times L$ sites $(L$ rows and $L$ columns) is used (see Fig. 1). The deposition process is performed with the following restrictions: (1) the $k$-mers contain $k$ identical units and each one occupies a lattice site, and small adsorbates with spherical symmetry would 


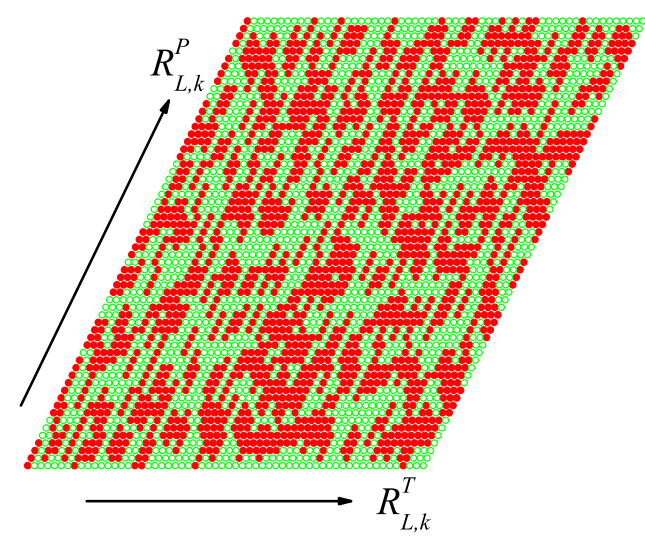

FIG. 1. Snapshot corresponding to a configuration of aligned tetramers $(k=4)$ on a rhombus-shaped triangular lattice. Open green circles and solid red circles represent empty sites and tetramer units, respectively.

correspond to the monomer limit $(k=1)$; (2) the distance between $k$-mer units is assumed in registry with the lattice constant $a$, and hence exactly $k$ sites are occupied by a $k$-mer when deposited; (3) the $k$-mers are deposited along one of the directions of the lattice, forming an oriented structure as depicted in Fig. 1; (4) the incoming particles must not overlap with previously added objects; and (5) periodic boundary conditions are considered.

Due to the blocking of the lattice by the already randomly adsorbed elements, the limiting or jamming coverage, $p^{j}=$ $p(t=\infty)$ is less than that corresponding to the close packing $\left(p^{j}<1\right)$. Note that $p(t)$ represents the fraction of lattice sites covered at time $t$ by the deposited objects. Consequently, $p$ ranges from 0 to $p^{j}$ for objects occupying more than one site [9]. For a fully aligned system, as studied here, the jamming problem reduces to the one-dimensional (1D) case. In this limit, an important exact result for jamming concentration $p^{j}(k)$ of linear $k$-mers in 1D RSA has been reported by Krapivsky et al. [11,26]:

$$
p^{j}(k)=k \int_{0}^{\infty} \exp \left[-u-2 \sum_{j=1}^{k-1}\left(\frac{1-e^{-j u}}{j}\right)\right] d u .
$$

As an example, the jamming coverage for dimers $(k=2)$ deposited along one line is $p^{j}(2)=1-e^{-2} \approx 0.864665$ and for trimers $(k=3)$ is $p^{j}(3)=3 D(2)-3 e^{-3} D(1) \approx$ 0.823653 , where $D(x)=e^{-x^{2}} \int_{0}^{x} e^{s^{2}} d s$ is Dawson's integral. For infinitely long rods $(k \rightarrow \infty)$, the jamming threshold tends to Rényi's parking constant $p^{j}(k \rightarrow \infty) \rightarrow c_{R} \approx$ 0.7475979202 [62]. Interested readers are referred to Ref. [11] (chap. 7) for a detailed description of Eq. (1).

\section{PERCOLATION}

\section{A. Calculation method and percolation thresholds}

As mentioned in Sec. I, the central idea of the pure percolation theory is based in finding the minimum concentration of elements (sites or bonds) for which a cluster extends from one side to the opposite one of the system. For this particular value of the concentration rate, the percolation threshold $p_{c}$, at least one spanning cluster (also called "infinite" cluster, inspired by the thermodynamic limit) connects the borders of the system [63-66]. In that case, a second-order phase transition appears at $p_{c}$, which is characterized by well-defined critical exponents.

In the simulations, each run consists of the following stages: (a) the construction of the lattice for the desired fraction $p=k N / M$ of sites ( $N$ is the number of $k$-mers deposited), according to the filling procedure presented in previous section and (b) the cluster analysis by using the Hoshen and Kopelman algorithm $[67,68]$ with open boundary conditions. In the last step, the size of the largest cluster $S_{L}$ is determined, as well as the existence of a percolating island. For this purpose, the probability $R=R_{L, k}^{X}(p)$ that a $L \times L$ lattice percolates at a concentration $p$ of sites occupied by rods of size $k$ can be defined. Here the following definitions can be given according to the meaning of $X[1,69,70]$ :

$R_{L, k}^{P}(p)$ : the probability of finding a percolating cluster in a parallel direction to the alignment axis (see Fig. 1)

$R_{L, k}^{T}(p)$ : the probability of finding a percolating cluster in a transverse direction to the alignment axis (see Fig. 1).

Other useful definitions for the finite-size analysis are the following:

$R_{L, k}^{U}(p)$ : the probability of finding either a parallel or a transverse percolating cluster

$R_{L, k}^{I}(p)$ : the probability of finding a cluster which percolates both in a parallel and in a transverse direction and

$$
R_{L, k}^{A}(p)=\frac{1}{2}\left[R_{L, k}^{U}(p)+R_{L, k}^{I}(p)\right] .
$$

$n$ runs of such two steps are carried out for obtaining the number $m^{X}$ of them for which a percolating cluster of the desired criterion $X$ is found. Then $R_{L, k}^{X}(p)=m^{X} / n$ is defined, and the procedure is repeated for different values of $p, L$, and $k$. A set of $n=10^{6}$ independent samples is numerically prepared for each pair $p$ and $L / k(L / k=75,100,128,256$ and 640). The $L / k$ ratio is kept constant to prevent spurious effects due to the $k$-mer size in comparison with the lattice linear size $L$.

In Fig. 2 the probabilities $R_{L, k}^{A}(p), R_{L, k}^{I}(p)$, and $R_{L, k}^{U}(p)$ are presented for aligned rods with $k=4$. As mentioned above, the simulations were performed for lattice sizes ranging between $L / k=75$ and $L / k=640$. Three sizes are shown in the figure: $L / k=128$ (up triangles), $L / k=256$ (down triangles), and $L / k=640$ (diamonds). Several conclusions can be drawn from Fig. 2 (and from data are not shown here for clarity). First, curves for different lattice sizes but with the same value of $k$ cross each other in a unique point, $R_{k}^{X^{*}}$ (measured in the vertical axis, see figure), which depends on the criterion $X$ used, and those points are located at very well-defined values in the $p$ axes determining the critical percolation threshold (measured on the horizontal axis; see figure) for each $k$. Second, $p_{c}(k)$ shifts to the right upon increasing the $k$-mer size. This observation is a clear indication that the percolation threshold increases upon increasing $k$.

Third, $R_{k}^{I^{*}}$ and $R_{k}^{U^{*}}$ show a strong dependence on the $k$ mer size. For $k=1$ (data not shown here), $R_{1}^{I^{*}} \approx 0.311$ and $R_{1}^{U^{*}} \approx 0.687$, as reported in previous work for standard site percolation on a rhombus-shaped lattice with open boundary conditions [71]. As $k$ is increased, two well-differentiated behavior are observed: (1) $R_{k}^{U^{*}}$ increases monotonically to $R_{k}^{U^{*}} \approx 1$ for larger sizes and (2) $R_{k}^{I^{*}}$ decreases monotonically 


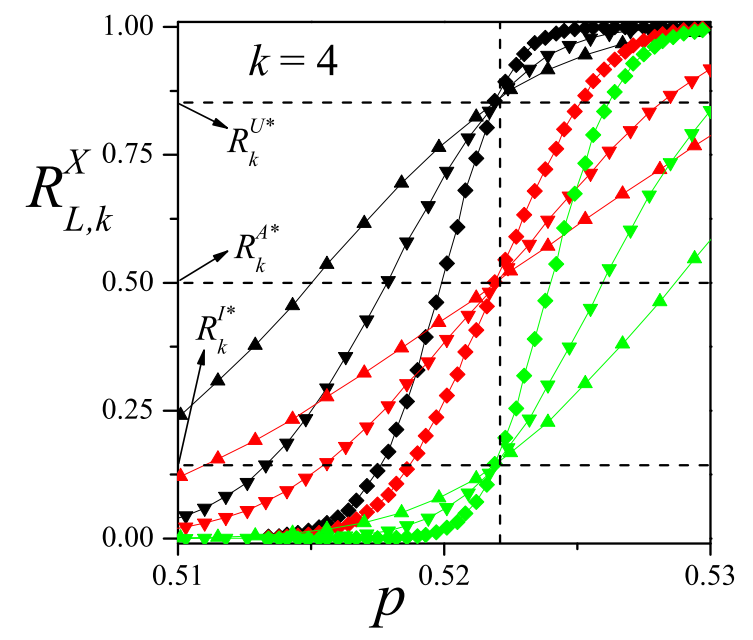

FIG. 2. Fraction of percolating lattices $R_{L, k}^{X}(p)(X=I, U, A$ as indicated) as a function of the concentration $p$ for $k=4$ and three different lattice sizes: $L / k=128$ (up triangles), $L / k=256$ (down triangles), and $L / k=640$ (diamonds). The vertical dashed line denotes the percolation threshold in the thermodynamic limit.

to $R_{k}^{I^{*}} \approx 0$ for larger sizes. On the other hand, $R_{k}^{A^{*}}$ remains constant (around 0.5 [71]) when $k$ increases. A similar behavior has been observed in the case of aligned $k$-mers on square lattices [55] and thermal transitions in the presence of anisotropy [72,73].

In percolation theory, the value of the probability $R_{L}^{X}$ at the transition point in the thermodynamic limit plays an important role in the scaling theory, being indicative of the universality class of the transition. From this perspective, the dependence of $R_{k}^{I^{*}}$ and $R_{k}^{U^{*}}$ on $k$ could be taken as a first indication of a nonuniversal behavior of the system for variable $k$-mer size. However, as pointed out by Selke et al. [72,73], the measure of the probability intersection may depend on various details of the model which do not affect the universality class, in particular, the boundary condition, the shape of the lattice, and the anisotropy of the system. Consequently, more research is required to determine the universality class of the phase transition.

In order to express $R_{L, k}^{X}(p)$ as a function of continuous values of $p$, it is convenient to fit $R_{L, k}^{X}(p)$ with some approximating function through the least-squares method. The fitting curve is the error function because $d R_{L, k}^{X} / d p$ is expected to behave like the Gaussian distribution [74]

$$
\frac{d R_{L, k}^{X}}{d p}=\frac{1}{\sqrt{2 \pi} \Delta_{L, k}^{X}} \exp \left\{-\frac{1}{2}\left[\frac{p-p_{c}^{X}(L, k)}{\Delta_{L, k}^{X}}\right]^{2}\right\},
$$

where $p_{c}^{X}(L, k)$ is the concentration at which the slope of $R_{L, k}^{X}(p)$ is the largest and $\Delta_{L, k}^{X}$ is the standard deviation from $p_{c}^{X}(L, k)$.

The standard procedure just described is valid for $R_{L, k}^{T}(p)$ and $R_{L, k}^{P}(p)$ in all range of $k$. The same does not occur in the case of $R_{L, k}^{A}(p)$. In fact, as will be discussed in detail later (see Figs. 7 and 8 below), the anisotropy of the percolating phase leads to a separation between the parallel and transversal probabilities. As a consequence of this separation, which

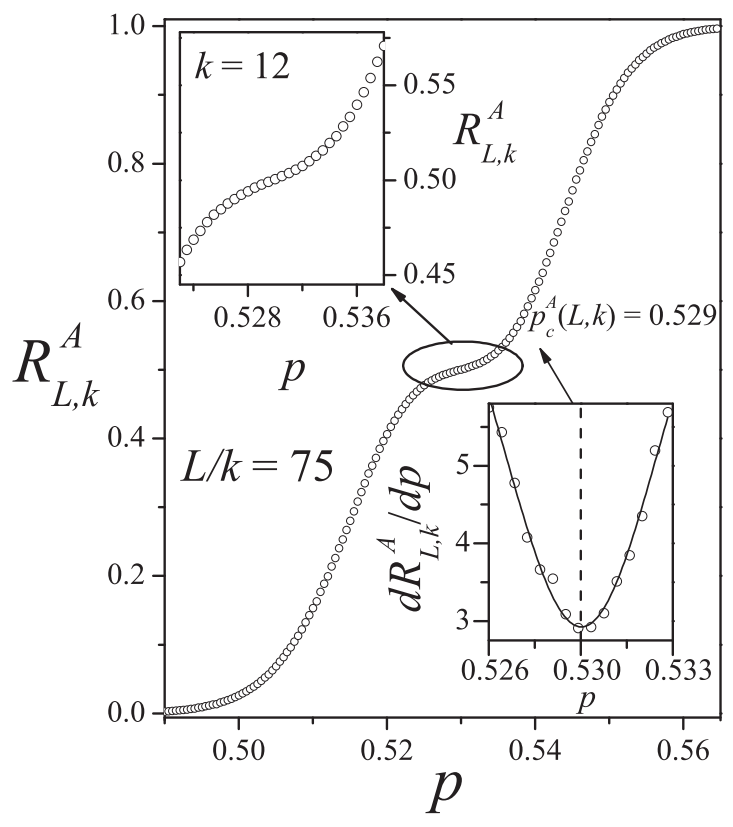

FIG. 3. Percolation probability $R_{L, k}^{A}(p)$ as a function of the concentration $p$ for $k=12$ and $L / k=75$. Upper-left inset: Magnification of the main figure in the range $0.523 \leqslant p \leqslant 0.538$. Lower-right inset: $d R_{L, k}^{A} / d p$ as a function of $p$ around the critical point $p_{c}^{A}(L, k)$. Symbols correspond to simulation data, and the solid line represents a Gaussian fitting curve.

increases with $k$, the $R_{L, k}^{A}(p)$ curves tend to gradually develop a plateau, with a marked inflection point around $R_{k}^{A^{*}} \approx 0.5$. This inflection point, which is barely perceptible in Fig. 2, can be clearly visualized in Fig. 3, where the percolation probability $R_{L, k}^{A}(p)$ has been plotted as a function of the concentration $p$ for $k=12$ and $L / k=75$.

The upper-left inset shows a zoom of the plateau region. On the other hand, the lower-right inset shows $d R_{L, k}^{A} / d p$ as a function of $p$ around the inflection point. Thus, the value of $p_{c}^{A}(L, k)$ can be obtained from the concentration at which the minimum occurs. For an accurate determination of this concentration, we fit the simulation data with an inverted Gaussian function. The procedure is shown in the lower-right inset: open circles correspond to simulation data, and the solid line represents the Gaussian fitting curve.

Once we have determined the positions $p_{c}^{X}(L, k)$, the percolation threshold $p_{c}(k)$ can be obtained using an extrapolation scheme. Thus, for each criterion $(I, U$, and $A)$, and for each value of $k$, one expects that [1]

$$
p_{c}^{X}(L, k)=p_{c}(k)+A^{X} L^{-1 / v},
$$

where $A^{X}$ is a nonuniversal constant and $v$ is the critical exponent of the correlation length. As will be shown in Sec. III B, the values of the critical exponents characterizing the percolation phase transition occurring in our model are consistent with those of 2D random percolation [75]: $v=4 / 3$, $\gamma=43 / 18$, and $\beta=5 / 36$ [1].

Figure 4 shows the plots towards the thermodynamic limit of $p_{c}^{X}(L, k)(X \equiv P, T, I, A, U)$ according to Eq. (3) for the cases $k=4$ [Fig. 4(a)] and $k=8$ [Fig. 4(b)]. From extrapolations it is possible to obtain the percolation thresholds for 


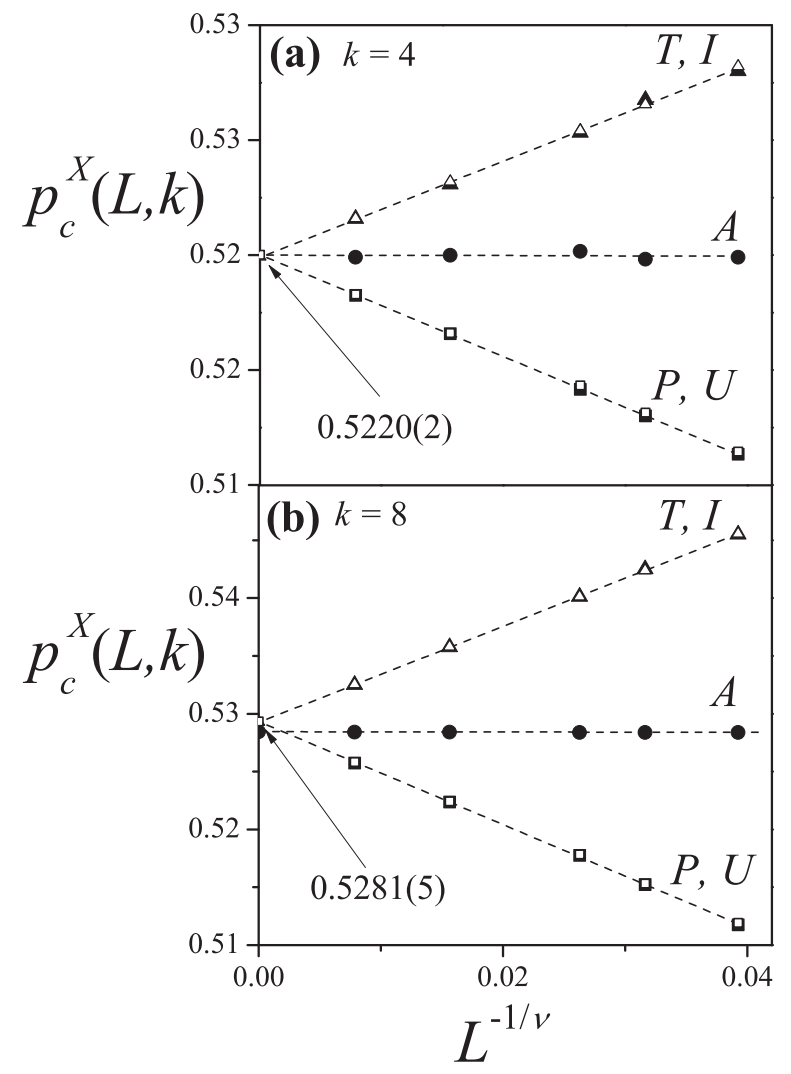

FIG. 4. Extrapolation of $p_{c}^{X}(L, k)$ towards the thermodynamic limit according to the theoretical prediction given by Eq. (3). Open squares, open triangles, solid triangles, solid circles, and solid squares denote the values of $p_{c}^{X}(L, k)$ obtained by using the criteria $P, T, I, A$, and $U$, respectively. The data correspond to the cases $k=4$ (a) and $k=8$ (b).

the criteria $I, A$, and $U$. Combining the three estimates for each case, the final values of $p_{c}(k)$ can be obtained. Additionally, the maximum of the differences between $\mid p_{c}(k)^{U}-$ $p_{c}(k)^{A} \mid$ and $\left|p_{c}(k)^{I}-p_{c}(k)^{A}\right|$ gives the error bar for each determination of $p_{c}(k)$. In this case, the values obtained were $p_{c}(k=4)=0.5220(2)$ [Fig. 4(a)] and $p_{c}(k=8)=$ $0.5281(5)$ [Fig. 4(b)].

It is interesting to analyze the behavior of $p_{c}^{P}(L, k)$ (open squares) and $p_{c}^{T}(L, k)$ (open triangles). As can be observed from Fig. 4, the curves corresponding to $P$ and $U$ criteria are coincident. The same goes for $T$ and $I$ criteria. This behavior can be easily understood by noticing that $p_{c}^{P}(L, k)<p_{c}^{T}(L, k)$ (fixed and finite $L)$. However, as $L \rightarrow \infty\left(L^{-1 / v} \rightarrow 0\right)$, both quantities converge to the same limit value $p_{c}^{P}(k)=p_{c}^{T}(k)=$ $p_{c}(k)$. This point will be discussed in detail in regard to Figs. 7 and 8 below.

The study shown in Figs. 2-4 demonstrates the relevance of the probability $R_{L, k}^{A}(p)$ for calculating percolation thresholds. In fact, we can clearly observe in Fig. 2 the point at which $R_{L, k}^{A}(p)=0.5$ behaves like the so-called fixed point. This fact supports that, even when the system size $L$ is not very large, $p_{c}^{A}(L, k)$ is very close to the threshold $p_{c}(k)$ of an infinite lattice [let us note that $p_{c}^{A}(L, k)$ gives a perfect horizontal line in Figs. 4(a) and 4(b)]. Such a behavior has been already reported in the literature [69].
TABLE I. Percolation thresholds versus $k$.

\begin{tabular}{lc}
\hline \hline$k$ & $p_{c}(k)$ \\
\hline 2 & $0.5157(2)$ \\
4 & $0.5220(2)$ \\
8 & $0.5281(5)$ \\
12 & $0.5298(8)$ \\
16 & $0.5328(7)$ \\
32 & $0.5407(6)$ \\
48 & $0.5455(4)$ \\
64 & $0.5487(8)$ \\
80 & $0.5500(6)$ \\
\hline \hline
\end{tabular}

The procedure in Fig. 4 was repeated for different values of $k$ ranging between 2 and 80 . The obtained values of $p_{c}(k)$ are collected in Table I (second column) and are plotted in Fig. 5 (open squares). As can be observed from the figure, the percolation threshold increases upon increasing $k$. The curve rapidly increases for small values of $k$, then it flatten out for larger values of $k$ and asymptotically converge towards a definite value as $k \rightarrow \infty$. In the range $2 \leqslant k \leqslant 80$, the data of $p_{c}(k)$ can be fitted with the function proposed in Ref. [25]: $p_{c}(k)=A+B /(C+\sqrt{k})$, where $A=p_{c}(k \rightarrow \infty)=0.582(9)$ the value of the percolation threshold by infinitely long $k$-mers, $B=-0.47(0.21)$, and $C=5.79(2.18)$. The adjusted coefficient of determination is $R^{2}=0.9899$. As observed in previous theoretical [41-44],

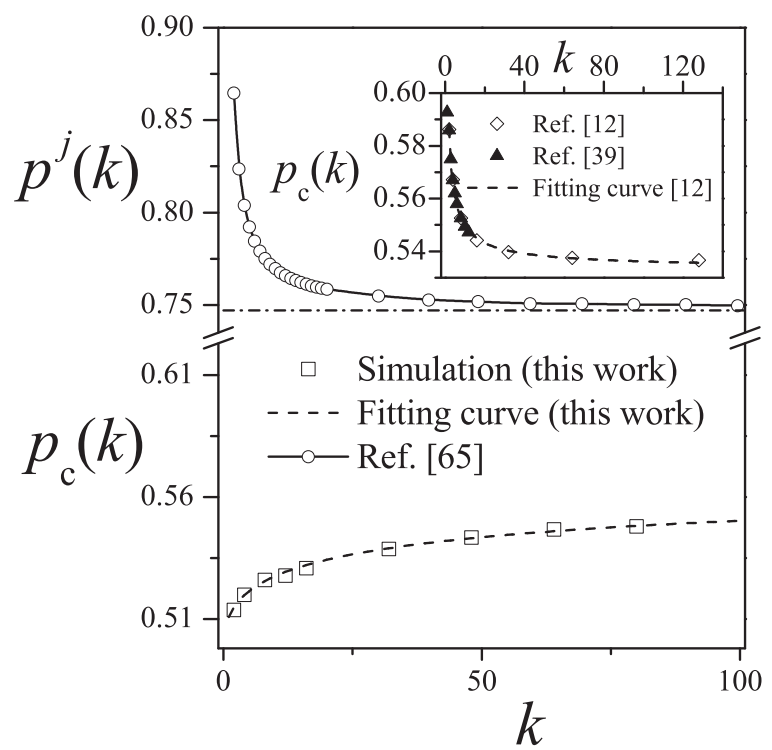

FIG. 5. Percolation threshold as a function of $k$ for aligned $k$ mers on triangular lattices (open squares). In all cases, the error bar is smaller that the size of the symbols. The dashed line corresponds to the fitting function $p_{c}(k)=A+B /(C+\sqrt{k})$ (see discussion in the text). The figure also includes the curve of $p^{j}(k)$ (open circles joined by a solid line). The corresponding numerical values were obtained by solving Eq. (1) (with $t \rightarrow \infty$ ). Inset: Percolation threshold as a function of $k$ for aligned $k$-mers on square lattices. Solid triangles and open diamonds denote previous data in Refs. [55] and [21], respectively. The dashed line represents the fitting curve obtained in Ref. [21]: $p_{c}(k)=a_{1} / k^{\alpha_{1}}+p_{c}(k \rightarrow \infty)$. 


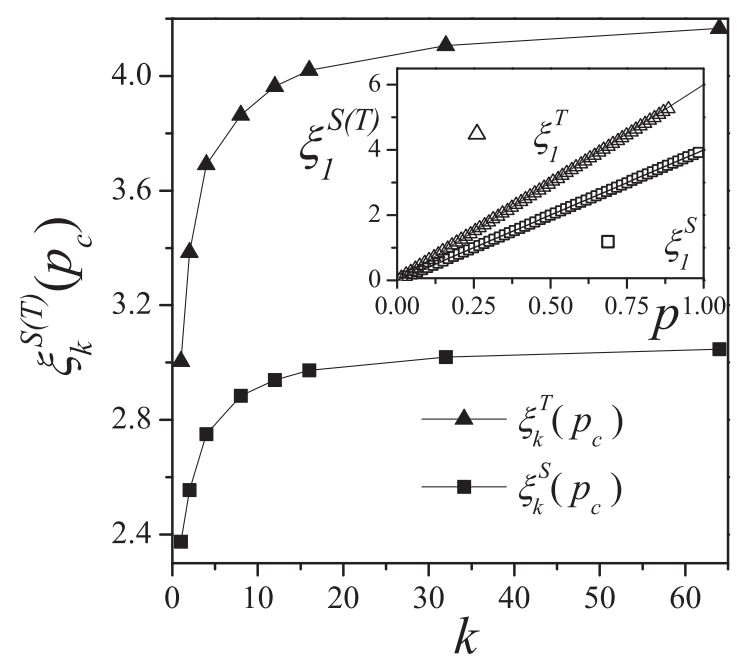

FIG. 6. Connectivity of the deposited phase at percolation threshold $\xi_{k}^{(T) S}\left(p_{c}\right)$ as a function of $k$. Open squares and open triangles represent results obtained for square and triangular lattices, respectively. In all cases, $L / k=100$. Inset: Average number of occupied nearest-neighbor sites $\xi_{k}^{S(T)}$ as a function of the surface coverage $p$ for $L=100$ and $k=1$. The solid lines correspond to theoretical curves as discussed in the text.

experimental [76-78], and simulation work [21,55,79-81], the percolation threshold is higher for aligned rods than for rods isotropically deposited (see Ref. [82], where the problem of isotropic $k$-mers on triangular lattices has been studied).

The inset of Fig. 5 shows the behavior of $p_{c}(k)$ for aligned $k$-mers on square lattices. Solid triangles and open diamonds correspond to data in Refs. [55] and [21], respectively. The dashed line represents the fitting curve obtained in Ref. [21]: $p_{c}(k)=a_{1} / k^{\alpha_{1}}+p_{c}(k \rightarrow \infty)$, where $p_{c}(k \rightarrow$ $\infty)=0.533(1), a_{1}=0.088(3)$, and $\alpha_{1}=0.72(4)$. These results are qualitatively different from those obtained for triangular lattices (main figure). Clearly, the structure of the lattice plays a fundamental role in determining the statistics and percolation properties of extended objects.

To gain some understanding on the observed differences between square and triangular percolation results, the connectivity properties of the aligned $k$-mers were analyzed. Namely, for each geometry (square and triangular) and each value of $k$, the average number of occupied nearest-neighbor sites of each occupied site (normalized to the lattice size $M$ ) was measured as a function of the lattice coverage $p$. We denote these functions as $\xi_{k}^{S}(p)$ and $\xi_{k}^{T}(p)$ for square and triangular lattices, respectively.

It is useful to begin the study with the case $k=1$, where we can go beyond numerical simulations. Thus, considering that the monomers are distributed completely at random on the lattice, $\xi_{k=1}^{S}(p)$ can be written as $\xi_{k=1}^{S}(p)=4 p$, where 4 is the square lattice connectivity and $p$ is the occupation probability of each site. As expected, the corresponding curve varies between $\xi_{k=1}^{S}=0(p=0)$ and $\xi_{k=1}^{S}=4(p=1)$. In the case of triangular lattices, $\xi_{k=1}^{T}(p)=6 p$, where $\xi_{k=1}^{T}=0$ $(p=0)$ and $\xi_{k=1}^{T}=6(p=1)$. See the inset in Fig. 6, where symbols and lines correspond to simulation data for $L=100$ and theoretical curves, respectively. For $k>1$, the statistical problem becomes more complex, and it is difficult to obtain analytically the functions $\xi_{k}^{S(T)}(p)$.

We will focus now on the behavior of the connectivity of the deposited phase (clusters of occupied sites) at percolation threshold $\xi_{k}^{(T) S}\left(p_{c}\right)$. In the case $k=1, \xi_{1}^{S}\left(p_{c} \approx 0.5927\right) \approx$ 2.3708 and $\xi_{1}^{T}\left(p_{c} \approx 0.5\right) \approx 3.0 . \xi_{k}^{(T) S}\left(p_{c}\right)$ as a function of $k$ is shown in Fig. 6 for square (solid squares) and triangular (solid triangles) lattices with $L / k=100$ and different values of $k$ ranging from $k=1$ to $k=64$. The behavior is qualitatively similar for square and triangular lattices: the curves increase monotonically with the increase of the size $k$, varying between $\xi_{1}^{(T) S}\left(p_{c}\right)(k=1)$ and a limiting value at large sizes $k$. These limit values are $\xi_{k \rightarrow \infty}^{S} \approx 3.1$ and $\xi_{k \rightarrow \infty}^{T} \approx 4.2$.

The results shown in Fig. 6 indicate that, in terms of connectivity, the structure of the percolating phase at the critical condition tends to be similar as $k$ is increased. This finding is found to be independent of lattice geometry (square or triangular), validating the tendencies observed for $p_{c}(k)$ in Fig. 5. However, further research is necessary to fully understand the differences between the curves of $p_{c}(k)$ for square and triangular lattices. Along this line, future efforts will be devoted (1) to extend the simulations to honeycomb lattices and higher values of $k$, (2) to analyze the connectivity of the percolation cluster, and (3) to compare the simulation data with theoretical results obtained from exact counting of configurations on finite cells [83].

Returning to Fig. 5, the analysis also includes the behavior of $p^{j}(k)$ for aligned $k$-mers (open circles joined by a solid line). The corresponding numerical values were obtained by solving Eq. (1). For this purpose, any standard mathematical software package (such as MAPLE or MATHEMATICA) can be used. It is also possible to measure $p^{j}(k)$ from simulations. The corresponding procedure is described by García et al. [84]. The study in Ref. [84] includes an exhaustive comparison between simulation results and exact values obtained from Eq. (1).

As it can be observed from Fig. 5, the curve of $p^{j}(k)$ remains above the curve of $p_{c}(k)$, tending to $p^{j}(k \rightarrow \infty) \approx$ 0.7475979202 in the limit of infinitely long rods [62]. This finding indicates that the RSA model of aligned $k$-mers on triangular lattices presents percolation transition in the whole range of $k$.

To complete the study, and given the anisotropy of the percolating phase, it is interesting to analyze the behavior of the transversal $\left[R_{L, k}^{T}(p)\right]$ and parallel $\left[R_{L, k}^{P}(p)\right]$ percolation probabilities. In Fig. 7 the probabilities $R_{L, k}^{P}(p)$ (solid symbols) and $R_{L, k}^{T}(p)$ (open symbols) are presented for a typical case: aligned rods with $k=4$ and different lattice sizes between $L / k=75$ and $L / k=640$. From a simple inspection of the figure it is observed that, first, for a fixed value of $L=L_{1}$, the $R_{L_{1}, k}^{P}(p)$ curve shifts to the left of the $R_{L_{1}, k}^{T}(p)$ curve. The result indicates that for finite systems the anisotropy of the deposited layer favors the percolation along the direction of the alignment axis. This scenario does not occur in isotropic systems, where for a fixed $L$, the vertical and horizontal percolation probabilities are indistinguishable [85]. Then, second, $R_{k}^{P^{*}}$ and $R_{k}^{T^{*}}$ crossing points are located at the same point on the $p$ axis (vertical line in the figure), indicating that, in the thermodynamic limit [86], the value 


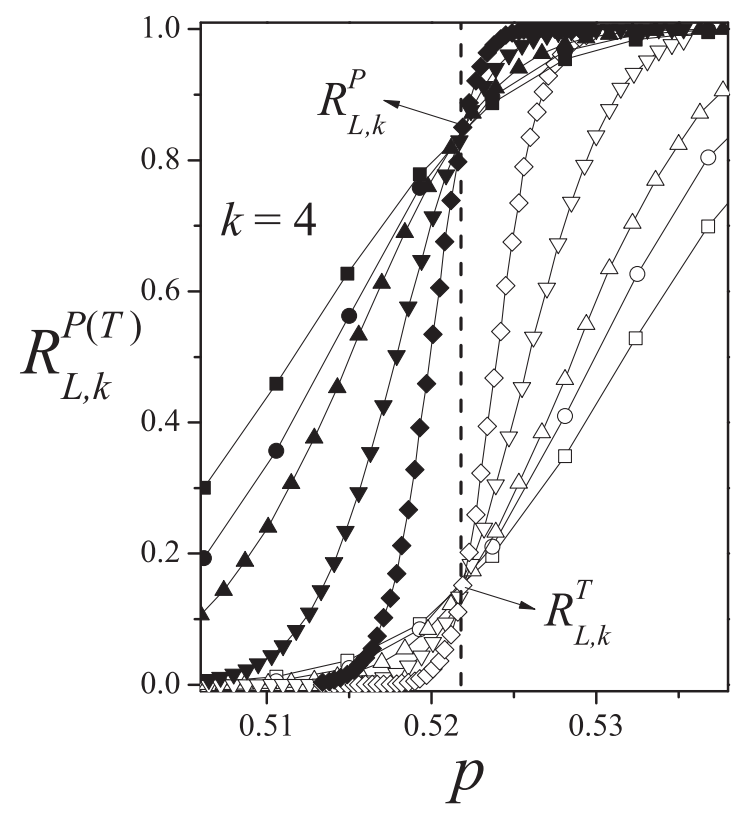

FIG. 7. Fraction of percolating lattices $R_{L, k}^{X}(p)(X=P, T$ as indicated) as a function of the concentration $p$ for $k=4$ and different lattice sizes: $L / k=75$ (squares), $L / k=100$ (circles), $L / k=128$ (up triangles), $L / k=256$ (down triangles), and $L / k=640$ (diamonds). The vertical dashed line denotes the percolation threshold in the thermodynamic limit.

of the percolation threshold is the same in both parallel and transversal directions.

An alternative way to visualize the effects described in the two points that we have made above is presented in Fig. 8. In Fig. 8(a) the parallel and transversal probabilities are shown for $k=4$ and a fixed value of the lattice size $L / k=128$. In order to measure the separation between the curves in the $p$ space, the distance $\delta(L, k)$ is defined as $\delta(L, k)=p_{T}^{*}(L, k)-$ $p_{P}^{*}(L, k)$, where $p_{T[P]}^{*}(L, k)$ is the value of the concentration $p$ for which $R_{L, k}^{T[P]}(p)=0.5$.

$\delta(L, k)$ was calculated for different values of $k$ and $L$. The results are shown in Fig. 8(b) for two $k$ sizes $(k=4$ and 8$)$ and $L / k$ ranging between 75 and 640 (note the log-log scale in the figure). In all cases, the separation between the parallel and transversal curves diminishes for increasing $L$, where $\delta(L \rightarrow \infty, k)=0$. This result reinforces the arguments given in the discussion of Fig. 7, that, namely, for an infinite system of aligned $k$-mers on triangular lattices, the properties of the percolating phase are characterized by a unique percolation threshold, regardless of the lattice direction (transversal or parallel to the alignment direction).

\section{B. Critical exponents and universality}

In this section the critical exponents $\nu, \beta$, and $\gamma$ will be calculated. Knowing $\nu, \beta$, and $\gamma$ is enough to determine the universality class of our system and understand the related phenomena.

In addition to the different probabilities $R_{L, k}^{X}(\theta)$, the percolation order parameter $P$, the susceptibility $\chi$, and the reduced fourth-order cumulant $U$, will be used to obtain the critical
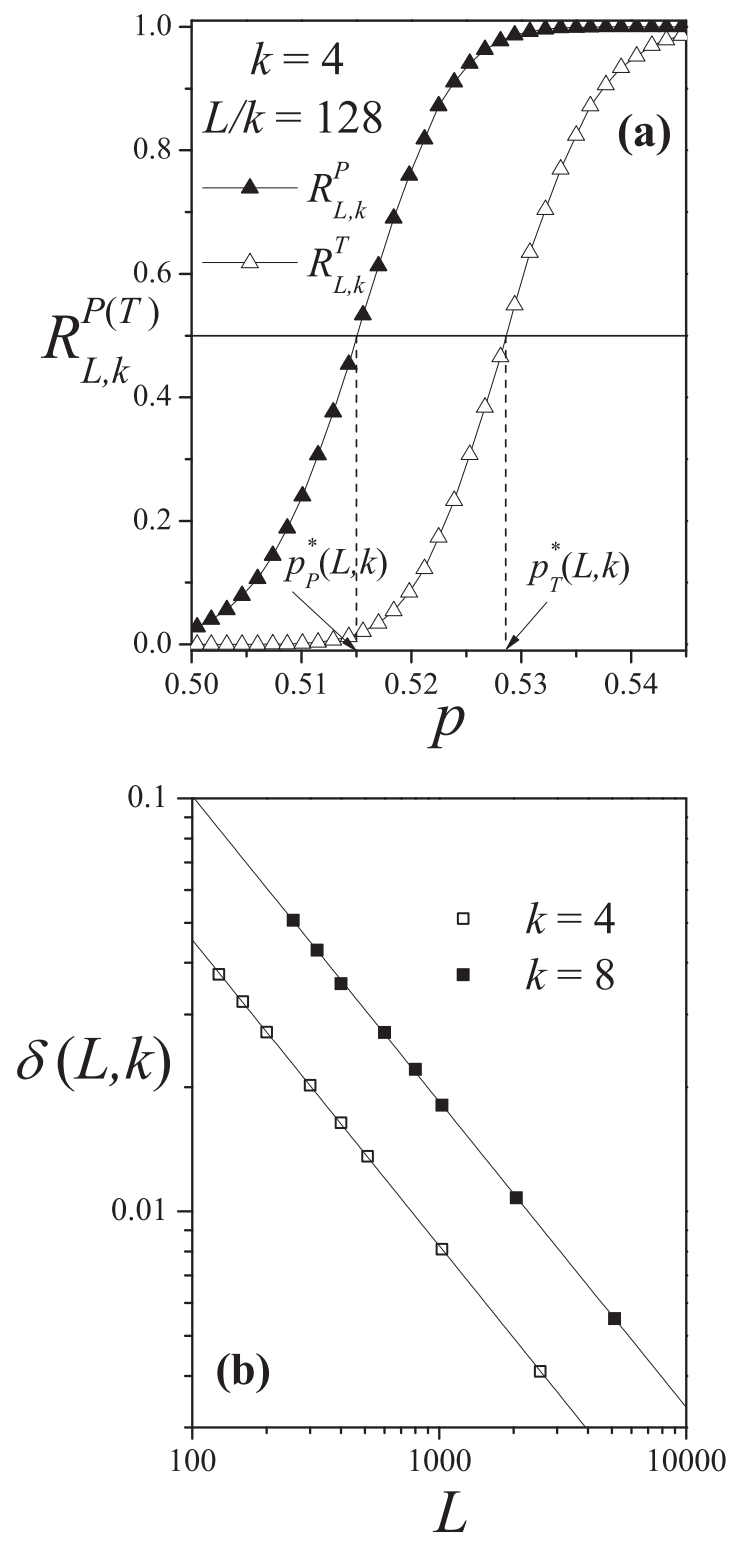

FIG. 8. (a) $R_{L, k}^{P}(p)$ (solid triangles) and $R_{L, k}^{T}(p)$ (open triangles) as a function of the concentration $p$ for $k=4$ and $L / k=128$. We show the procedure used to measure the distance $\delta(L, k)$ (see discussion in the text). (b) Log-log plot of $\delta(L, k)$ as a function of the lattice size $L / k$ for two different values of $k: k=4$, open squares, and $k=8$, solid squares.

exponents. These quantities can be calculated as

$$
\begin{gathered}
P=\frac{\left\langle S_{L}\right\rangle}{M}, \\
\chi=\frac{\left[\left\langle S_{L}^{2}\right\rangle-\left\langle S_{L}\right\rangle^{2}\right]}{M},
\end{gathered}
$$

and

$$
U=1-\frac{\left\langle S_{L}^{4}\right\rangle}{3\left\langle S_{L}^{2}\right\rangle^{2}},
$$

where $S_{L}$ represents the size of the largest cluster, and $\langle\cdot\rangle$ means an average over simulation runs. 

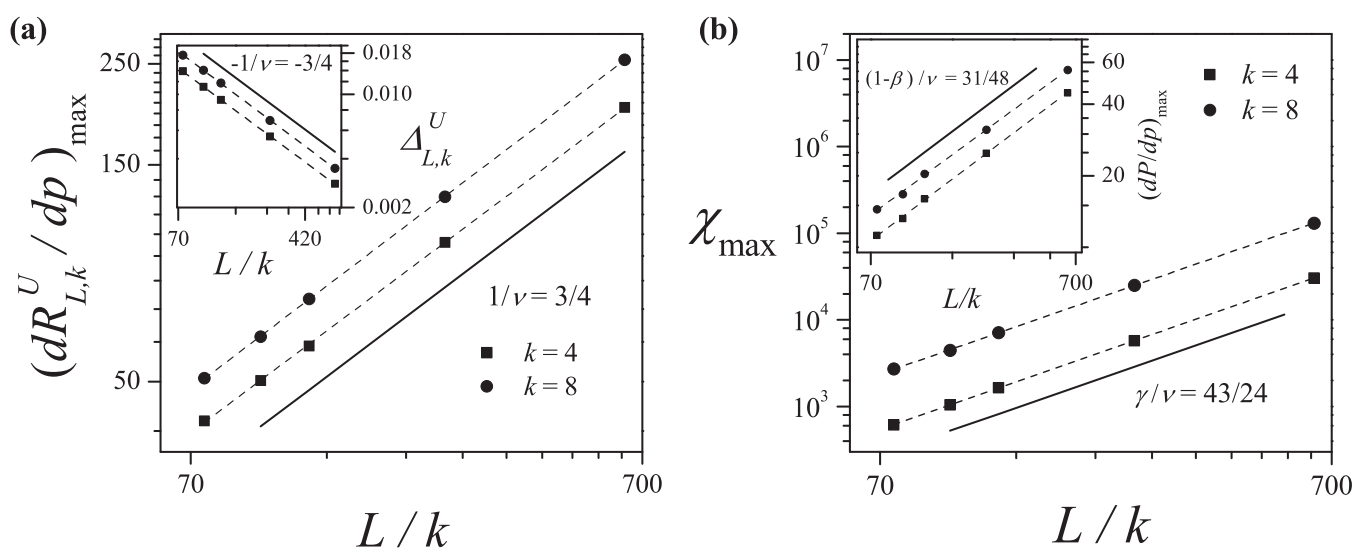

FIG. 9. (a) Maximum of the derivative of the $U$ percolation probability $\left(d R_{L, k}^{U} / d p\right)_{\max }$ as a function of $L / k$ (in a log-log scale) for two different cases: $k=4$ (solid squares) and $k=8$ (solid circles). The error bar in each measurement is smaller than the size of the symbols. According to Eq. (7) the slope of each line corresponds to $1 / v=3 / 4$. Inset: Standard deviation $\Delta_{L, k}^{U}$ as a function of $L / k$ (in a log-log scale) for the same cases shown in the main figure. According to Eq. (8), the slope of each line corresponds to $-1 / v=-3 / 4$. (b) Maximum of the susceptibility $\chi_{\max }$ as a function of $L / k$ (in a log-log scale) for two different cases: $k=4$ (solid squares) and $k=8$ (solid circles). The error bar in each measurement is smaller than the size of the symbols. The slope of each line corresponds to $\gamma / v=43 / 24$. Inset: Maximum of the derivative of the percolation order parameter $(d P / d p)_{\max }$ as a function of $L / k$ (in a log-log scale) for the same cases reported in the main figure. According to Eq. (9), the slope of each line corresponds to $(1-\beta) / v=31 / 48$.

The standard theory of finite size [87] allows for various routes to estimate the critical exponent $v$ from simulation data. One of these methods is from the maximum of the function $d R_{L, k}^{X} / d p$ :

$$
\left(\frac{d R_{L, k}^{X}}{d p}\right) \propto L^{1 / \nu} .
$$

In Fig. 9(a) $\log \left[\left(d R_{L, k}^{U} / d p\right)_{\max }\right]$ has been plotted as a function of $\log [L]$ for $k=4$ and $k=8$. According to Eq. (7), the slope of each line corresponds to $1 / v$. As can be observed, the slopes of the curves remain constant, where $v=1.338(9)$ for case $k=4$ and $v=1.328$ (4) for case $k=8$.

Another alternative way for evaluating $v$ is from the divergence of the root-mean-square deviation of the percolation threshold observed from their average values, $\Delta_{L, k}^{X}$ :

$$
\Delta_{L, k}^{X} \propto L^{-1 / v}
$$

As an example of validity of Eq. (8), the inset in Fig. 9(a) shows $\Delta_{L, k}^{U}$ as a function of $L$ (note that both axis are in log$\log$ scale) for the same cases of the main figure. According to Eq. (8) the slope of the line corresponds to $-1 / v$. In this case, $v=1.335(6)$ for $k=4$ and $v=1.329(5)$ for $k=8$.

Once $v$ is known, the exponents $\gamma$ and $\beta$ were determined by the finite-size scaling theory [1]. On one hand, $\gamma$ is obtained by scaling the maximum value of the susceptibility $\chi$. According to scaling assumption for this quantity given by $\chi=L^{\gamma / v} \bar{\chi}(u)$, where $u=\left(p-p_{c}\right) L^{1 / v}$ and $\bar{\chi}$ is the corresponding scaling function. At the point where $\chi$ is maximal, $u=$ const, and $\chi_{\max } \propto L^{\gamma / \nu}$. Our simulation data are presented in Fig. 9(b). The values obtained are $\gamma=2.39$ (1) for $k=4$ and $\gamma=2.40$ (2) for $k=8$.

On the other hand, the exponent $\beta$ is calculated from the scaling behavior at criticality $P=L^{-\beta / \nu} \bar{P}\left(u^{\prime}\right)$, where $u^{\prime}=$ $\left|p-p_{c}\right| L^{1 / v}$ and $\bar{P}$ is the scaling function. At the point where $d P / d p$ is maximal, $u^{\prime}=$ const and

$$
\left(\frac{d P}{d p}\right)_{\max }=L^{(-\beta / v+1 / v)} \bar{P}\left(u^{\prime}\right) \propto L^{(1-\beta) / v} .
$$

The scaling tendencies for some particular cases are shown in the inset of Fig. 9(b). From the slopes of the curves, the following values of $\beta$ were obtained: $\beta=0.136$ (2) for $k=4$ and $\beta=0.138$ (3) for $k=8$.

The results in Fig. 9 agree, within numerical errors, with the exact values of $v=4 / 3, \gamma=43 / 18$, and $\beta=5 / 36$ for $2 \mathrm{D}$ ordinary percolation.

The scaling behavior has been tested by plotting $P L^{\beta / v}$ versus $\left|p-p_{c}\right| L^{1 / \nu}, \chi L^{-\gamma / v}$ versus $\left(p-p_{c}\right) L^{1 / \nu}, R_{L}^{A}$ versus $\left(p-p_{c}\right) L^{1 / \nu}$, and $U$ versus $\left(p-p_{c}\right) L^{1 / v}$ and looking for data collapsing. Using the values of $p_{c}$ obtained and the exact values of the critical exponents corresponding to ordinary
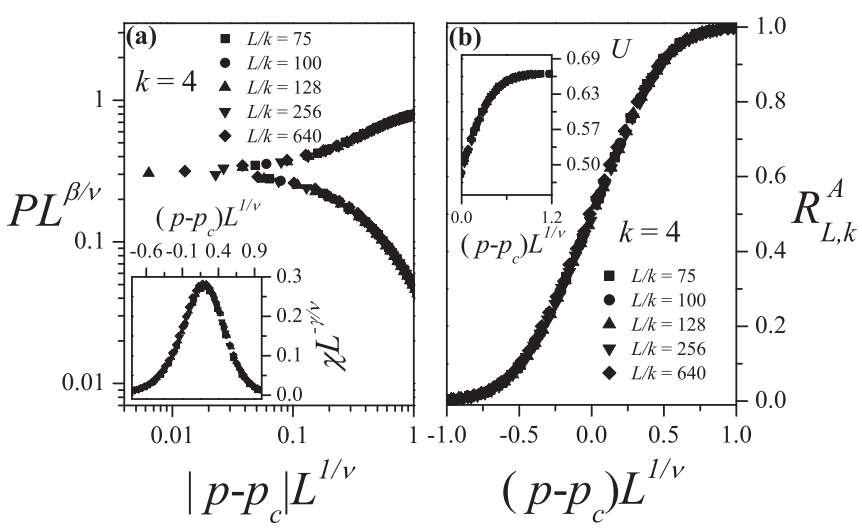

FIG. 10. (a) Data collapsing of the percolation order parameter, $P L^{\beta / v}$ vs $\left|p-p_{c}\right| L^{1 / v}$, and of the susceptibility, $\chi L^{-\gamma / v}$ vs $(p-$ $\left.p_{c}\right) L^{1 / v}$ (inset), for $k=4$. (b) Data collapsing of the percolation probability, $R_{L, k}^{A}(p)$ vs $\left(p-p_{c}\right) L^{1 / v}$, and the cumulant, $U_{L}$ vs $(p-$ $\left.p_{c}\right) L^{1 / v}$ (inset), for $k=4$. 
percolation $v=4 / 3, \gamma=43 / 18$, and $\beta=5 / 36$, the curves have an excellent scaling collapse; see Figs. 10(a) and 10(b).

It is well known that RSA has very short-range correlations, which are corroborated by the values of $\nu, \gamma$, and $\beta$ reported above. This also means that the percolation properties of the system presented here correspond to the same universality class as the random percolation problem.

\section{CONCLUSIONS}

In this paper, the percolation behavior of aligned rigid rods of length $k$ on 2D triangular (rhombus-shaped) lattices has been investigated by computer simulations and finite-size scaling analysis. The $k$-mers (with $k$ from 2 to 80 ) were deposited along one of the directions of the lattice, forming an oriented structure. Lattice sizes up to $L / k=640$ were used.

For each value of $k$, the size of the largest cluster $S_{L}$ and the probability $R_{L, k}^{X}(p)(X=P, T, U, I, A)$ that a lattice of size $L$ percolates at concentration $p$ were used to obtain the critical point (percolation threshold $p_{c}(k)$ and intersection point of the probability curves $R_{k}^{X^{*}}$ ) and the critical exponents $\nu, \beta$, and $\gamma$ characterizing the phase transition.

The percolation threshold exhibits a monotonic increasing function when it is plotted as a function of the $k$-mer size: $p_{c}(k)=A+B /(C+\sqrt{k})$, where $A=p_{c}(k \rightarrow \infty)=$ $0.582(9)$, the value of the percolation threshold by infinitely long $k$-mers, $B=-0.47(0.21)$, and $C=5.79(2.18)$. This behavior is completely different from that observed for square lattices, where the percolation threshold decreases with $k[21,55]$. The present result clearly demonstrates that the structure of the lattice plays a fundamental role in determining the statistics and percolation properties of extended objects. To finish with the analysis of $p_{c}(k)$, it is important to note that, for all $k$, the percolation threshold of aligned rods is higher than the corresponding one to isotropic $k$-mers [82].

The effect of the anisotropy on the properties of the percolating phase was investigated by following the behavior of $R_{L, k}^{P}(p)$ (probability of finding a percolating cluster in a parallel direction to the alignment axis) and $R_{L, k}^{T}(p)$ (probability of finding a percolating cluster in a transverse direction to the alignment axis). For finite systems, while in the case of isotropic $k$-mers the vertical and horizontal percolation probabilities are indistinguishable, in the case of aligned $k$-mers the anisotropy of the deposited layer favors the percolation along the direction of the alignment axis. The difference between the parallel and transversal probabilities diminishes for increasing the lattice size $L$, where $R_{L, k}^{P}(p)=R_{L, k}^{T}(p)$ in the limit of $L \rightarrow \infty$. In other words, the value of the percolation threshold is the same in both parallel and transversal directions.

The breaking of the orientational symmetry also influences the behavior of the percolation probabilities at the intersection point $R_{k}^{X^{*}}$. Thus, $R_{k}^{U^{*}}$ and $R_{k}^{I^{*}}$ exhibit a nonuniversal critical behavior, varying continuously with changing the $k$-mer size. A similar scenario already has been reported in the case of aligned $k$-mers on square lattices $[21,55]$ and thermal transitions in the presence of anisotropy [72,73]. These results indicate that the universality of the intersection points $R_{k}^{X^{*}}$ 's is observed only for isotropic systems. For anisotropic systems, this universality is violated, and the value of the crossing point of the percolation probabilities is dependent upon $k$ (and the degree of alignment).

Finally, the improved accuracy in the determination of the critical exponents $(\nu, \beta$, and $\gamma$ ) confirmed that the model belongs to the same universality class as the random percolation, regardless of the size $k$ considered. In addition, the corresponding curves collapse according to the predictions of the scaling theory.

\section{ACKNOWLEDGMENTS}

This work was supported in part by CONICET (Argentina) under project No. PIP 112-201101-00615, Universidad Nacional de San Luis (Argentina) under project No. 03-0816, and the National Agency of Scientific and Technological Promotion (Argentina) under project No. PICT-2013-1678. The numerical work was done using the BACO parallel cluster [88] located at Instituto de Física Aplicada, Universidad Nacional de San Luis-CONICET, San Luis, Argentina.
[1] D. Stauffer and A. Aharony, Introduction to Percolation Theory (Taylor \& Francis, London, 2003).

[2] M. Sahimi, Applications of Percolation Theory (Taylor \& Francis, London, 1994); Flow and Transport in Porous Media and Fractured Rock (VCH, Weinheim, Germany, 1995).

[3] G. Grimmett, Percolation (Springer-Verlag, Berlin, 1999).

[4] B. Bollobás and O. Riordan, Percolation (Cambridge University Press, New York, 2006).

[5] S. Kundu and S. S. Manna, Phys. Rev. E 93, 062133 (2016).

[6] S. Kundu, N. A. M. Araújo, and S. S. Manna, Phys. Rev. E 98, 062118 (2018).

[7] L. L. Treffenstädt, N. A. M. Araújo, and D. de las Heras, Soft Matter 14, 3572 (2018).

[8] J. Feder, J. Theoret. Biol. 87, 237 (1980).

[9] J. W. Evans, Rev. Mod. Phys. 65, 1281 (1993).

[10] J. Talbot, G. Tarjus, P. R. Van Tassel, and P. Viot, Colloid. Surface A 165, 287 (2000).
[11] P. L. Krapivsky, S. Redner, and E. Ben-Naim, A Kinetic View of Statistical Physics (Cambridge University Press, Cambridge, 2010).

[12] Z. Adamczyk, J. Barbasz, and M. Cieśla, Langmuir 26, 11934 (2010).

[13] Z. Adamczyk, J. Barbasz, and M. Cieśla, Langmuir 27, 6868 (2011).

[14] Z. Adamczyk, Curr. Opin. Colloid Interface Sci. 17, 173 (2012).

[15] M. Rabe, D. Verdes, and S. Seeger, Adv. Colloid Interface Sci. 162, 87 (2011).

[16] M. Cieśla, Z. Adamczyk, J. Barbasz, and M. Wasilewska, Langmuir 29, 7005 (2013).

[17] C. Finch, T. Clarke, and J. J. Hickman, J. Comput. Phys. 244, 212 (2013).

[18] Y. Pan, L. Iorga, and A. A. Pelegri, Computat. Mater. Sci. 43, 450 (2008). 
[19] J. Zhou, L. Qi, and A. M. Gokhale, ASME J. Eng. Mater. Technol. 138, 021001 (2016).

[20] Also denoted as needles, rods, or sticks.

[21] Y. Y. Tarasevich, N. I. Lebovka, and V. V. Laptev, Phys. Rev. E 86, 061116 (2012).

[22] Y. Y. Tarasevich, V. V. Laptev, N. V. Vygornitskii, and N. I. Lebovka, Phys. Rev. E 91, 012109 (2015).

[23] P. M. Centres and A. J. Ramirez-Pastor, J. Stat. Mech. (2015) P10011.

[24] G. Kondrat, Z. Koza, and P. Brzeski, Phys. Rev. E 96, 022154 (2017).

[25] M. G. Slutskii, L. Y. Barash, and Y. Y. Tarasevich, Phys. Rev. E 98, 062130 (2018).

[26] N. I. Lebovka, N. N. Karmazina, Y. Y. Tarasevich, and V. V. Laptev, Phys. Rev. E 84, 061603 (2011).

[27] J.-C. Charlier, X. Blase, and S. Roche, Rev. Mod. Phys. 79, 677 (2007).

[28] A. G. N. Sofiah, M. Samykano, K. Kadirgama, R. V. Mohan, and N. A. C. Lah, Appl. Mater. Today 11, 320 (2018).

[29] D. S. Hecht, L. Hu, and G. Irvin, Adv. Mater. 23, 1482 (2011).

[30] D. McCoul, W. Hu, M. Gao, V. Mehta, and Q. Pei, Adv. Electron. Mater. 2, 1500407 (2016).

[31] R. M. Mutiso and K. I. Winey, Prog. Polym. Sci. 40, 63 (2015).

[32] S. De, P. J. King, P. E. Lyons, U. Khan, and J. N. Coleman, ACS Nano 4, 7064 (2010).

[33] R. M. Mutiso, M. C. Sherrott, A. R. Rathmell, B. J. Wiley, and K. I. Winey, ACS Nano 7, 7654 (2013).

[34] T. Ackermann, R. Neuhaus, and S. Roth, Sci. Rep. 6, 34289 (2016).

[35] A. Kumar and G. U. Kulkarni, J. Appl. Phys. 119, 015102 (2016).

[36] A. Kumar, N. S. Vidhyadhiraja, and G. U. Kulkarni, J. Appl. Phys. 122, 045101 (2017).

[37] R. Taherian, Compos. Sci. Technol. 123, 17 (2016).

[38] S. P. Finner, M. I. Kotsev, M. A. Miller, and P. van der Schoot, J. Chem. Phys. 148, 034903 (2018).

[39] S. Torquato, Random Heterogeneous Materials, Interdisciplinary Applied Mathematics Vol. 16 (Springer, New York, 2002).

[40] G. E. Pike and C. H. Seager, Phys. Rev. B 10, 1421 (1974).

[41] I. Balberg and S. Bozowski, Solid State Commun. 44, 551 (1982).

[42] I. Balberg, N. Binenbaum, and C. H. Anderson, Phys. Rev. Lett. 51, 1605 (1983).

[43] I. Balberg, C. H. Anderson, S. Alexander, and N. Wagner, Phys. Rev. B 30, 3933 (1984).

[44] A. P. Chatterjee, J. Chem. Phys. 140, 204911 (2014).

[45] Y. Y. Tarasevich, V. A. Goltseva, V. V. Laptev, and N. I. Lebovka, Phys. Rev. E 94, 042112 (2016).

[46] N. I. Lebovka, Y. Y. Tarasevich, N. V. Vygornitskii, A. V. Eserkepov, and R. K. Akhunzhanov, Phys. Rev. E 98, 012104 (2018).

[47] Y. Y. Tarasevich, N. I. Lebovka, I. V. Vodolazskaya, A. V. Eserkepov, V. A. Goltseva, and V. V. Chirkova, Phys. Rev. E 98, 012105 (2018).

[48] A. V. Kyrylyuk and P. van der Schoot, Proc. Natl. Acad. Sci. USA 105, 8221 (2008).

[49] R. H. J. Otten and P. van der Schoot, Phys. Rev. Lett. 103, 225704 (2009).
[50] R. H. J. Otten and P. van der Schoot, J. Chem. Phys. 134, 094902 (2011).

[51] R. H. J. Otten and P. van der Schoot, Phys. Rev. Lett. 108, 088301 (2012).

[52] T. Schilling, M. A. Miller, and P. van der Schoot, Europhys. Lett. 111, 56004 (2015).

[53] S. Kwon, H. W. Cho, G. Gwon, H. Kim, and B. J. Sung, Phys. Rev. E 93, 032501 (2016).

[54] S. P. Finner, T. Schilling, and P. van der Schoot, Phys. Rev. Lett. 122, 097801 (2019).

[55] P. Longone, P. M. Centres, and A. J. Ramirez-Pastor, Phys. Rev. E 85, 011108 (2012).

[56] Lj. Budinski-Petković and U. Kozmidis-Luburić, Phys. Rev. E 56, 6904 (1997).

[57] Lj. Budinski-Petković, M. Petković, Z. M. Jakšić, and S. B. Vrhovac, Phys. Rev. E 72, 046118 (2005).

[58] I. Lončarević, Lj. Budinski-Petković, and S. B. Vrhovac, Phys. Rev. E 76, 031104 (2007).

[59] Lj. Budinski-Petković, I. Lončarević, Z. M. Jakšić, S. B. Vrhovac, and N. M. Švrakić, Phys. Rev. E 84, 051601 (2011).

[60] Lj. Budinski-Petković, I. Lončarević, M. Petković, Z. M. Jakšić, and S. B. Vrhovac, Phys. Rev. E 85, 061117 (2012).

[61] Lj. Budinski-Petković, I. Lončarević, Z. M. Jakšić, and S. B. Vrhovac, J. Stat. Mech. (2016) 053101.

[62] A. Rényi, Sel. Transl. Math. Stat. Probab. 4, 203 (1963) [translation from Magyar Tud. Akad. Mat. Kutató Int. Közl. 3, 109 (1958)].

[63] M. Aizenman, Nucl. Phys. B 485, 551 (1997).

[64] J. Cardy, J. Phys. A 31, L105 (1998).

[65] L. N. Shchur and S. S. Kosyakov, Int. J. Mod. Phys. C 8, 473 (1997).

[66] L. N. Shchur, in Computer Simulation Studies in CondensedMatter Physics XII, Springer Proceedings in Physics Vol. 85, edited by D. P. Landau, S. P. Lewis, and H. B. Schuettler (Springer Verlag, Heidelberg, 2000), pp. 129-146.

[67] J. Hoshen and R. Kopelman, Phys. Rev. B 14, 3438 (1976).

[68] J. Hoshen, R. Kopelman, and E. M. Monberg, J. Stat. Phys. 19, 219 (1978).

[69] F. Yonezawa, S. Sakamoto, and M. Hori, Phys. Rev. B 40, 636 (1989).

[70] F. Yonezawa, S. Sakamoto, and M. Hori, Phys. Rev. B 40, 650 (1989).

[71] L. S. Ramirez, N. De la Cruz Félix, P. M. Centres, and A. J. Ramirez-Pastor, J. Stat. Mech. (2017) 023206.

[72] W. Selke and L. N. Shchur, J. Phys. A: Math. Gen. 38, L739 (2005).

[73] W. Selke, J. Stat. Mech. (2007) P04008.

[74] Even though the behavior of $d R_{L, k}^{X} / d p$ is known not to be a Gaussian in all range of $p$, this quantity is approximately Gaussian near the peak, and Eq. (2) is a good approximation for the purpose of locating its maximum [85].

[75] The results about the universality class are expected since it is well understood that without long-range correlations, the percolation universality class will not change. It is also well known that RSA has very short-range correlations.

[76] F. Du, J. E. Fischer, and K. I. Winey, Phys. Rev. B 72, 121404(R) (2005).

[77] N. Grossiord, J. Loos, O. Regev, and C. E. Koning, Chem. Mater. 18, 1089 (2006). 
[78] H. Deng, R. Zhang, E. Bilotti, J. Loos, and T. Peijs, J. Appl Polym. Sci. 113, 742 (2009).

[79] S. S. Rahatekar, M. Hamm, M. S. P. Shaffer, and J. A. Elliott, J. Chem. Phys. 123, 134702 (2005).

[80] S. I. White, B. A. DiDonna, M. Mu, T. C. Lubensky, and K. I. Winey, Phys. Rev. B 79, 024301 (2009).

[81] S. Kale, F. A. Sabet, I. Jasiuk, and M. Ostoja-Starzewski, J. Appl. Phys. 120, 045105 (2016).

[82] E. J. Perino, D. A. Matoz-Fernandez, P. M. Pasinetti, and A. J. Ramirez-Pastor, J. Stat. Mech. (2017) 073206.
[83] W. Lebrecht, E. E. Vogel, J. F. Valdés, A. J. Ramirez-Pastor, P. M. Centres, M. I. González, and F. D. Nieto, Phys. Rev. E 92 , 012129 (2015).

[84] G. D. García, F. O. Sanchez-Varretti, P. M. Centres, and A. J. Ramirez-Pastor, Physica A 436, 558 (2015).

[85] M. E. J. Newman and R. M. Ziff, Phys. Rev. Lett. 85, 4104 (2000).

[86] The thermodynamic limit for infinitely long rods is obtained by doing $L \rightarrow \infty, k \rightarrow \infty$ and $L / k=$ finite.

[87] K. Binder, Rep. Prog. Phys. 60, 488 (1997).

[88] http://cluster_infap.unsl.edu.ar/wordpress/ 